- Bei Beschlagnahme verbal Widerstand äußern, Helfen beim Heraussuchen ist aber möglich, Kopien der Unterlagen erstellen!

_Sicherstellungsverzeichnis aushändigen lassen und schriftlich widersprechen.

\section{Eizellspende im Ausland}

Nimmt eine Frau die in Deutschland verbotene Eizellspende im Ausland in
Anspruch, ist dies nicht strafbar, für den mitwissenden und bei der Vorbereitung helfenden Gynäkologen aber möglicherweise nach $₫ 1$ Embryonenschutzgesetz wegen Anstiftung und Beihilfe schon. Medizinrechtlerin Dr. Dorothea Magnus aus Hamburg rät:

— Bei Verdacht auf eine Eizellspende bei der Patienten genau nach dem Zweck der gewünschten Hormontherapie fragen,
_Mithilfe zur Vorbereitung der Eizellspende ablehnen,

- keine direkten Empfehlungen für Zentren im Ausland geben.

Die weitere Betreuung der nach Eizellspende Schwangeren im Sinne der Mutterschaftsrichtlinien ist juristisch kein Problem.

Friederike Klein

6. Hauptthema „Rechtliche Brennpunkte in der Gynäkologie", 5.3.2016

\title{
Empfehlungen zur Pessartherapie bei Deszensus und Inkontinenz
}

$E^{\mathrm{i}}$ ine Therapie eines Deszensus oder einer Inkontinenz - egal ob chirurgisch oder konservativ - ist nur indiziert, wenn die betroffenen Frauen unter den Beschwerden leiden, betonte Dr. Jacek Kocieszewski vom Kontinenz- und Beckenbodenzentrum Hagen-Witten. Am Anfang einer konservativen Therapie steht die Beratung hinsichtlich der Intimpflege und die Östrogenisierung der Vulva und Vagina. „Die Hälfte der Inkontinenzsymptome geht dann oft schon weg", ist Kocieszewskis Erfahrung. Die lokale Applikation sollte auch erfolgen, wenn die Frau parallel eine hormonelle Kontrazeption oder eine transdermale oder orale HRT anwendet. In den ersten vier Behandlungswochen empfiehlt er eine Östriolcreme vaginal (es reiche auch das Eincremen von Vulva und Scheideneingang), danach einbis zweimal pro Woche abends als Langzeittherapie, an den übrigen Tagen der Woche eine milchsäurehaltige Creme. Eine deutliche Verbesserung der Atrophie - und oft auch der Symptome - ist erstmals meist nach sechs Wochen spürbar. Wird eine Östrogencreme absolut abgelehnt, können beispielsweise mit einer wasserfreien Intimpflegesalbe einmal täglich Vulva und Scheideneingang gut eingefettet werden.

Verhaltenstherapeutisch sollte zur Gewichtsabnahme und zum Verzicht auf Noxen wie Nikotin geraten werden - leider bleibe dies oft ohne Erfolg.

Eine wichtige Basis für die weitere Therapie ist die physiotherapeutische Stärkung des Beckenbodens. „Das gilt auch für die chirurgische Therapie“, betonte Kocieszewski. Gerade ältere Frauen benötigen hier Anleitung, um in Zukunft bei Husten, Aufstehen und anderen Belastungen bewusst die vulnerablen Strukturen entlasten zu können. Spezialisierte Therapeuten finden sich im Internet (z. B. bei der AG GGUP - Gynäkologie Geburtshilfe Urologie Proktologie im Deutschen Verband für Physiotherapie ZVK e.V., www.ag-ggup.de).

Eine Pessartherapie setzt voraus, dass die Frauen regelmäßig zur Kontrolle kommen. Kontraindikationen sind aktive vaginale Infektionen, Infektionen des kleinen Beckens und vaginale Blutungen unklarer Ursache.

Das bei Inkontinenz angewendete Urethrapessar ist ring- oder schalenförmig und liegt auf den Levatormuskeln auf, diese müssen daher intakt sein. Die eigentliche Funktion hängt von der korrekten Lage der Pellote ab: Sie muss mittig vor der Harnröhre anliegen, darf sie aber nicht abdrücken. Damit kann ein Urinverlust verringert oder auch komplett verhindert werden. Urethraschalenpessare liegen besser an als Ringpessare, sagte Kocieszewski.

Würfelpessare haften an der Vaginalhaut und sind daher von der Levatorenfunktion unabhängig bei einer Senkung anwendbar. Die Anpassung erfolgt hier beginnend mit einer relativ großen Größe hin zu kleineren Würfeln. Die Pessare können durch kontinuierliche Stimulation des Gewebes den Beckenboden soweit stärken, dass immer kleinere Pessare nötig sind und schließlich wieder ganz darauf verzichtet werden kann. Vor Beginn dieser Therapie sollte man darauf hinweisen, dass die Behandlung unter Umständen eine durch den Organvorfall maskierte Inkontinenz zutage fördern kann. Diese müsse dann entsprechend behandelt werden.

Würfelpessare mit Löchern erzeugen weniger Ausfluss, weil sich weniger Flüssigkeit staut, haften aber weniger gut als solche ohne Löcher. Modelle mit Fäden sollen die Entfernung erleichtern. Aber Vorsicht: immer erst den angesaugten Würfel vorsichtig lösen und zusammendrücken, nicht einfach herausziehen, empfiehlt Kocieszewski.

Die Anpassung der Pessare sollte immer unter der Voraussetzung erfolgen, dass eine Atrophie über einige Wochen mit Östrogen lokal behandelt worden ist. „Setzen Sie daher ein Pessar niemals beim ersten Mal ein“, riet Kocieszewski, "sonst will die Frau nie wieder eines." Optimal angepasst ist das Pessar, wenn die Frau nichts spürt. Ist es zu groß, kann es Schmerzen verursachen und die Schleimhaut verletzen, ist es zu klein, verrutscht es oder fällt heraus. „65 mm ist ein guter Durchschnittswert bei deutschen Frauen“, definierte Kocieszewski.

Nach der Anpassung sollte die erste Kontrolle nach ein bis zwei Wochen erfolgen, Kocieszewski empfiehlt dann bei täglichem eigenhändigem Wechsel weitere Kontrollen nach drei Monaten und dann halbjährlich, bei Wechsel des Pessars durch den Arzt alle acht Wochen. Bei jedweden Beschwerden sollten Patientinnen immer sofort den Arzt aufsuchen.

Friederike Klein

Kurs "Moderne Pessartherapie bei Deszensus und Harninkontinenz in der Praxis", 5.3.2016 